\title{
EVALUACIÓN DE PRODUCTOS BOTÁNICOS Y QUÍMICOS PARA EL CONTROL DE MOSCA BLANCA (Bemisia tabaci, Gennadius.) Y GEMINIVIRUS EN EL CULTIVO DE TOMATE (Solanum esculentum, Mill.), EN TISMA, MASAYA
}

\section{EVALUATION OF BOTANICAL AND CHEMICAL INSECTICIDES TO CONTROL WHITEFLY (Bemisia tabaci, Gennadius.) AND GEMINIVIRUS IN TOMATO (Solanum esculentum, Mill.), IN TISMA, MASAYA}

Jiménez-Martínez Edgardo', Lanuza Rodríguez Eliezer Hazael², Rizo González Edwin José2. ${ }^{1} \mathrm{PhD}$. en Entomología, Universidad Nacional Agraria, Profesor Titular, Docente-Investigador.

${ }^{2}$ Ing. en Sistemas de Protección Agrícola y forestal, UNA.

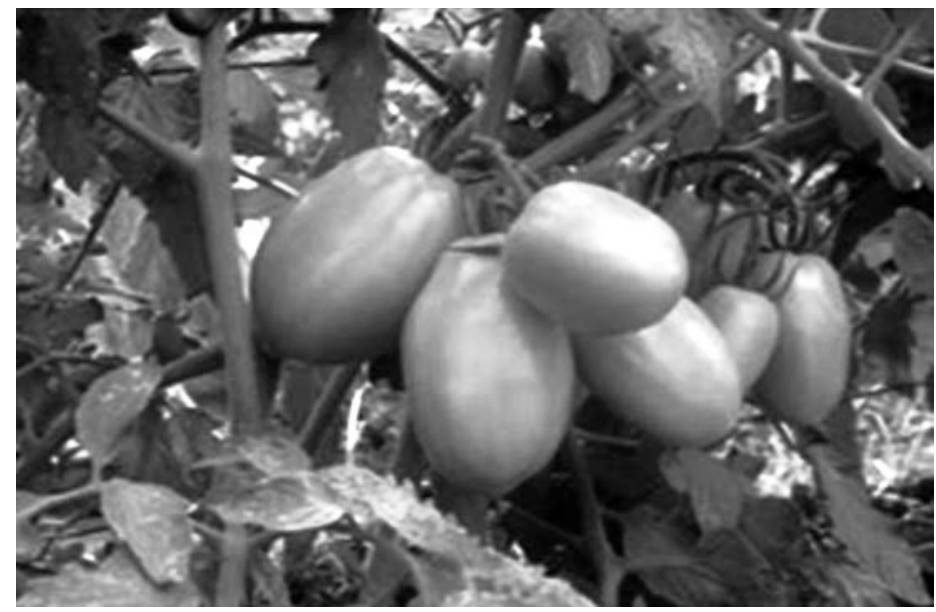

\section{RESUMEN}

La mosca blanca (Bemisia tabaci, Genn) y los Geminivirus son los principales agentes de daño causantes de problemas fitosanitarios severos para los productores de tomate (Solanum esculentum L = Lycopersicum esculentum, Mill), en el municipio de Tisma, Masaya. Esta plaga provoca importantes pérdidas económicas, disminuyendo así los rendimientos al afectar la calidad de los frutos, lo cual incurre en mayores costos de producción. En base a esta problemática en el municipio de Tisma se realizó un estudio en el período comprendido de noviembre 2010 a enero 2011, con el objetivo de evaluar insecticidas botánicos y químicos contra el complejo mosca blanca-Geminivirus. Los productos evaluados fueron: Engeo, Imidacloprid, Crisantemo, Madero Negro, Chile+Ajo+Jabón. Las variables evaluadas fueron: número de mosca blanca por planta, incidencia y severidad del daño de virosis por planta. De los tratamientos evaluados, el menor promedio de moscas blancas por planta lo presentó el tratamiento Imidacloprid seguido de Crisantemo. El menor porcentaje de incidencia y severidad lo presentó el tratamiento Imidacloprid en comparación con los demás tratamientos. El análisis económico realizado determinó que los tratamientos que presentaron los mejores rendimientos fueron Crisantemo con $32578 \mathrm{~kg} \mathrm{ha}^{-1}$, seguido por Engeo con $31750 \mathrm{~kg} \mathrm{ha}^{-1}$ y Chile+Ajo+Jabón que obtuvo 30 $625 \mathrm{~kg} \mathrm{ha}^{-1}$. Crisantemo presentó el mayor beneficio neto con 7 546.90 US\$ ha ${ }^{-1}$ seguido por Engeo con 7254.58 US\$ ha- $^{-1}$. En el análisis de la tasa de retorno marginal resultó que el tratamiento Engeo es el que obtuvo la mejor tasa de retorno marginal con $8671.80 \%$ es decir 86.71 US\$ por cada dólar invertido. Palabras clave: Tomate, Mosca blanca, Geminivirus, Botánicos.

\section{ABSTRACT}

The whitefly (Bemisia tabaci Genn) and Geminiviruses are the main agents causing severe phytosanitary damages for tomato (Lycopersicon esculentum, Mill) producers in Tisma, Masaya. This pest complex cause economic losses, thereby reducing yields by affecting fruit quality, which incurs in higher production costs. Based on this problem, in Tisma, Masaya, an experiment was conducted in the period from November 2010 to January 2011. The objective of this experiment was to evaluate botanical and chemical insecticides against whitefly-geminivirus complex. The evaluated insecticides were: Chrysanthemum, Madero Negro, Chili + Garlic + Soap, Engeo and Imidacloprid. The evaluated variables were: number of whiteflies per plant, incidence and severity of viral damage per plant. Main results indicate that Imidacloprid followed by Chrysanthemum had the lowest number of whitefly per plant. Imidacloprid had the lowest incidence and severity rates, compared evaluated treatments. The economic analysis determined that the treatments with the highest yields were Chrysanthemum with $32578 \mathrm{~kg} \mathrm{ha}^{-1}$, followed by Engeo with $31750 \mathrm{~kg} \mathrm{ha}^{-1}$ and Chili+Garlic+Soap with $30625 \mathrm{~kg} \mathrm{ha}^{-1}$. Chrysanthemum had the highest net income with U.S. \$ 7 546.90/ha followed by Engeo with 7254.58 US \$/ha. The marginal rate return analysis shows that Engeo had the highest marginal rate, obtaining 8,671.80\% which means that 86.71 U.S. \$ is obtained for every dollar invested. Keywords: Tomato, Whitefly, Geminivirus, Botanical. 
$\mathrm{E}$ 1 tomate (Solanum esculentum L. = Lycopersicum esculentum, Mill.), es originario del sur de América, específicamente de la región andina (Perú, Bolivia y Ecuador), aunque el centro de domesticación fue el sur de México y el norte de Guatemala donde existe el mayor grado varietal de la planta (INTA, 2004). Taxonómicamente el tomate pertenece a la familia, Solanáceae y a la especie: Lycopersicum sculentum, esta es una planta perenne de porte arbustivo que se cultiva como anual, puede desarrollarse de forma rastrera, semierecta o erecta. Existen variedades de crecimiento limitado e indeterminado (Rayo, 2001). En Nicaragua, en el año 1970, se observó en el municipio de Tisma, Masaya una enfermedad afectando al cultivo del tomate que se le atribuye a la presencia de la mosca blanca. Ya en los años 80 se reportaron daños en varios cultivos de importancia alimenticia causado por la virosis transmitida por B. tabaci (Rojas et al; 2000). Los primeros reportes del daño por virosis en el país se presentaron en 1986 en el valle de Sébaco; para 1990-1991 se reportaron disminuciones de rendimiento del 20 al $25 \%$. En el año 1991-1992 las pérdidas oscilaron entre el 30 al 100\% (Gómez, 1992).

Como todo cultivo, el tomate presenta problemas fitosanitarios de plagas las que aumentan los costos de producción debido al alto uso de insecticidas, sumado esto al uso de fertilizantes, fungicidas y herbicidas. Uno de los principales problemas en el municipio de Tisma ha sido el ataque severo del complejo mosca blanca (B. tabaci)Geminivirus, su alta plasticidad genética la ha convertido en una plaga difícil de manejar, se conocen 17 razas o biotipos de B. tabaci, de ellas al menos seis están en América (Brown, et al., 1995), Bemisia tabaci pertenece taxonómicamente a la clase insecta, orden Hemiptera, suborden Sternorrhyncha y familia Aleyrodidae (Caballero, 1996, Jiménez-Martínez, 2009). Los principales daños causados directamente al cultivo del tomate se caracterizan por succionar la savia e inyectar sustancias fitotóxicas a la planta; pero también por la transmisión de Geminivirus causante de enfermedades viróticas, lo que es capaz de devastar por completo una área determinada del cultivo, donde las etapas más críticas son las primeras semanas después de la germinación de la planta (Jarquín, 2004). Los geminivirus pertenecen a la familia Geminiviridae y se dividen en cuatro géneros que se caracterizan según el vector que lo transmite, el hospedero y la estructura genómica que posee. Estos son: Mastrevirus, Curtovirus, Topocovirus y Begomovirus; este último es transmitido por la mosca blanca (Zúñiga y Ramírez, 2002).

Con el propósito de buscar alternativas de manejo a la problemática del ataque severo de mosca blanca-Geminivirus al cultivo del tomate en el municipio de Tisma, se estableció un experimento en el que se evaluaron productos botánicos usados por productores y productos químicos disponibles en el mercado. El propósito del estudio fue conocer el efecto que tienen los productos evaluados sobre el complejo mosca blanca-Geminivirus y otros insectos plagas asociados al cultivo del tomate, determinar si el cultivo presenta buenos rendimientos y conocer la factibilidad de dichos productos, logrando de esta manera poner en mano de los productores nuevas tecnologías que ayuden a mantener a un nivel bajo las poblaciones de mosca blanca, de manera que se disminuyan las pérdidas económicas, los costos de producción, la contaminación de suelos y agua, las alteraciones a la cadena trófica y sobre todo cuidar la salud humana. Los objetivos de este estudio fueron: Evaluar cinco productos botánicos y químicos para el manejo del complejo mosca blancaGeminivirus en tomate, evaluar el efecto de productos botánicos y químicos sobre la ocurrencia poblacional de otros insectos plagas asociados al cultivo, comparar el rendimiento comercial en cada uno de los tratamientos evaluados para el manejo de mosca blanca-Geminivirus y comparar la factibilidad económica de cada tratamiento evaluado a través de un análisis económico de presupuesto parcial.

\section{MATERIALES Y MÉTODOS}

Ubicación del estudio. El estudio se realizó en el municipio de Tisma, departamento de Masaya en la finca El Chagüite propiedad de la señora Elizabeth González en el periodo comprendido entre los meses de octubre 2010 a enero 2011. Tisma está ubicada a $36 \mathrm{~km}$ de la ciudad de Managua capital de Nicaragua a una altura de $50 \mathrm{msnm}$ entre las coordenadas $12^{\circ} 04^{\prime}$ de latitud norte y $86^{\circ} 01^{\prime}$ de longitud oeste, se caracteriza por un clima tropical de sabana con temperatura de $27.5^{\circ} \mathrm{C}$ y precipitaciones pluviales anuales entre $1200 \mathrm{y}$ $1400 \mathrm{~mm}$ (AMUNIC, 2005).

Establecimiento del ensayo. Antes de realizar el ensayo, se estableció un semillero de tomate el 12 de octubre del 2010 bajo las condiciones de micro-invernadero. La variedad de tomate utilizada fue Shanty, esta variedad es del tipo Roma con alta tolerancia a TYLCV, fruto de 120 a 150 gramos de peso y de color rojo intenso con hombros claros y de larga vida de anaquel. Esta variedad es vigorosa, para cultivo de estaca y/o piso en campo abierto. Las plántulas se establecieron en bandejas de polietileno de 105 celdas, el sustrato utilizado fue cascarilla de arroz carbonizada y lombrihumus en una proporción de 50 y $50 \%$, donde se depositaron las semillas; estas fueron tratadas con el fungicida sistémico Phyton®. El Phyton es un fungicida, bactericida, sistémico, de amplio espectro de acción permitido en cultivos orgánicos. Se utilizó una dosis de $44 \mathrm{~cm}^{3}$ por cada 20 litros de agua.

Diseño experimental. El estudio se estableció bajo un diseño de bloques completos al azar (BCA) con cuatro repeticiones por tratamiento, donde se evaluaron tres tratamientos botánicos y dos químicos para el manejo de 
mosca blanca. Se utilizó un tamaño de parcela de $10 \mathrm{~m}$ de largo por $8 \mathrm{~m}$ de ancho para un área por parcela de $80 \mathrm{~m}^{2}$, para un total de área por bloque de $480 \mathrm{~m}^{2}$ y un área total del experimento de $1920 \mathrm{~m}^{2}$. Para el establecimiento de las plantas en la parcela se utilizaron distancias de siembra entre planta de $0.5 \mathrm{~m}$ y entre surco de un $\mathrm{m}$. Cada parcela estaba compuesta por ocho surcos y cada surco estaba conformado por un total de 20 plantas, para un total de 160 plantas por parcela dejando una separación entre parcela de $1.5 \mathrm{~m}$.

Muestreos de insectos. Para determinar el momento de la aplicación de los tratamientos se realizaron muestreos semanales por la mañana, para esto se seleccionaron los dos surcos centrales de cada parcela y se revisaban 25 plantas en los dos surcos centrales. Por cada planta se revisaban las hojas por el envés, los datos se anotaban en una hoja de registro de muestreo. Las variables evaluadas fueron: número de moscas blancas por planta, incidencia del daño de virosis, severidad del daño de virosis.

Aplicaciones de productos. Las aplicaciones de los productos se realizaron en base a los datos obtenidos en el muestreo, utilizando un nivel crítico poblacional de una mosca blanca por planta de tomate como parámetro de decisión para aplicar el tratamiento. Las aplicaciones se realizaron por aspersión directa al follaje haciendo uso de bomba de mochila con capacidad de 20 litros de agua y se efectuaron por las tardes para evitar deriva del producto.

\section{Descripción de los tratamientos}

Tratamiento 1: Engeo ${ }^{\circledR}$. (Thiametoxam $25 \%$ y lambdacihalotrina $10.6 \%$ ), para evaluar el tratamiento Engeo se utilizó una dosis de $10 \mathrm{~cm}^{3}$ por bombada de 20 litros de agua $\left(1 / 2 \mathrm{~cm}^{3}\right)$ por litro de agua. El engeo es un insecticida de amplio espectro de acción, actúa por contacto, por ingestión, y también posee efecto de repelencia y acción anti alimentaria.

Tratamiento 2: Imidacloprid ${ }^{\circledR}$. Es un formulado a base de imidacloprid, materia activa perteneciente al grupo químico de los cloronicotinilos. Para evaluar el tratamiento se utilizó una dosis de $21 \mathrm{~g}$ por bombada de capacidad de 201 . Este es un producto con propiedades insecticidas, su modo de acción lo realiza bloqueando los impulsos nerviosos de los insectos.

\section{Tratamiento 3: Crisantemo. (Chrysanthemum} cinerariaefolium Trevir), se utilizó una dosis de $450 \mathrm{~g}$ de producto molido lo cual incluye hojas y flores frescas por bomba de capacidad de 20 litros. Es insecticida, repelente, anti alimentario. Actúa por contacto-ingestión. El efecto insecticida se produce cuando las piretrinas naturales penetran al sistema nervioso del insecto, interfiriendo con el movimiento de iones de potasio $(\mathrm{K})$ y sodio $(\mathrm{Na})$, provocando en el insecto después de la aplicación, la pérdida de la capacidad de movilidad o vuelo.

Tratamiento 4: Madero negro. (Gliricidia sepium Jacq), La dosis utilizada fue de $450 \mathrm{~g}$ de producto macerado (hojas frescas) por bomba de 20 litros. Es un insecticida y abono foliar, su toxicidad se debe a la conversión por las bacterias de cumarinas a dicoumerol durante la fermentación. Se ha estudiado su actividad fagodisuasiva sobre los adultos de $B$. tabaci en condiciones de invernadero (Flores, et al., 2008).

Tratamiento 5: Chile Capsicum sp + Ajo Allium sativum + Jabón (Detergente). La dosis utilizada fue de $4 \mathrm{oz} \mathrm{de}$ chile molido más $4 \mathrm{oz}$ de ajo molidas más $2 \mathrm{oz}$ de jabón por bombada de 20 litros. El chile es insecticida y repelente, actúa inhibiendo la alimentación de los insectos. La capcicina (chile) actúa como ingrediente activo (Arrivillaga, et al., 1997).

Tratamiento 6: Testigo. Consistió solamente en aplicación de agua a las plantas una vez a la semana.

\section{Variables evaluadas}

Número de mosca blanca por planta. Esta variable se registró desde los 15 días después del trasplante (ddt), realizando muestreos semanales hasta los $93 \mathrm{ddt}$. Las plantas de tomate se muestreaban en su totalidad, específicamente en el envés de la hoja, lugar donde se encuentra más frecuentemente la mosca blanca.

Incidencia del daño de virosis por planta. La incidencia se refiere al número de plantas que presentan síntomas del daño por virosis transmitido por mosca blanca, usualmente relativo al número total de plantas evaluadas. La incidencia se estimó visualmente en las plantas que presentaban síntomas del daño de virosis asignándole un valor de cero a las plantas que no presentaban síntomas y uno a las que si presentaban síntomas. Para determinar la incidencia de virosis se registraron datos a partir de los 42 días después del trasplante (ddt) hasta los 93 ddt.

Para obtener el grado porcentual de la incidencia se utilizó la fórmula:

$$
\text { Incidencia }(\%)=\frac{\text { Total de plantas con síntomas virales }}{\text { Total de plantas muestreadas }} \times \mathbf{1 0 0}
$$

Severidad del daño de virosis por planta. La severidad es el porcentaje de tejido visualmente dañado o afectado de una planta con relación al total de biomasa por planta evaluada. Para poder diferenciar entre una planta sana de una enferma, la estimación se realizó a través de la observación de los síntomas característicos que presentan las hojas de las plantas 
atacadas por virosis: enanismo de la planta, mosaico en hojas nuevas o viejas y enrollado de hojas. Para determinar la severidad del daño de virosis se registraron datos desde los $42 \mathrm{ddt}$ hasta los 93 días después del trasplante (ddt).

Para obtener el grado porcentual de la severidad se utilizó la fórmula:

$$
\begin{aligned}
& \sum \text { de los grados de severidad } \\
& \text { Severidad }(\%)=\frac{\text { encontrados }}{\text { El número de plantas muestreadas } \mathrm{x}} \times 100 \\
& \text { el grado mas alto de la escala }
\end{aligned}
$$

Para determinar el grado de severidad ocasionado por mosca blanca se utilizó la escala de severidad propuesta por (Jiménez-Martínez, 2006), y luego modificada por (Jiménez-Martínez en el 2010).

Tabla 1. Escala de severidad de virosis modificada por (JiménezMartínez, 2010)

\begin{tabular}{ll}
\hline Escala & Características \\
\hline $0=0 \%$ & $\begin{array}{l}\text { No hay síntomas } \\
\text { Débil mosaico y encolochado en la lámina } \\
\text { foliar de las hojas nuevas } \\
\text { Mosaico y encolochado de las hojas } \\
\text { generalizado } \\
\text { Mosaico, encolochado y deformación de hojas }\end{array}$ \\
$3=75 \%$ & $\begin{array}{l}\text { sanas. } \\
\text { Enanismo y deformación severa }\end{array}$ \\
\hline
\end{tabular}

Rendimiento $\mathbf{~ g ~ h a ~}^{-1}$. Para obtener los datos de rendimiento por hectárea se midió el peso de los frutos maduros obtenido de 20 plantas por tratamiento, el rendimiento se registró en libras y posteriormente se transformó $\mathrm{kg} \mathrm{ha}^{-1}$.

Análisis de los datos. Una vez recolectados los datos en campo se procedió a ordenarlos por variable y luego hacer un análisis de varianza (ANDEVA). En las variables incidencia, severidad del daño de virosis, otros organismos plagas y rendimiento, se aplicó un ANDEVA analizado de forma unifactorial. En los casos que se encontró diferencia estadística se utilizó la separación de medias por Duncan $(\alpha=0.05)$. Previo al análisis de varianza se realizaron transformaciones de datos para ajustarlos a los supuestos del ANDEVA. Se utilizó $(y=\sqrt{x}+5)$ para incidencia y severidad $\left(\Theta=\operatorname{arcsen} \vee_{p}\right.$ ), por medio del (PROC GLM en SAS, 2003.V.9.1).

Análisis económico. Los resultados agronómicos que se obtuvieron del experimento de campo fueron sometidos a análisis económico con el propósito de determinar los tratamientos con mejor retorno económico. Los mejores tratamiento recomendados deben ajustarse a los objetivos y circunstancias de los productores (Alemán, 2004). Para determinar cuál de los tratamientos fue el más rentable tomando en cuenta la relación beneficio-costo, se realizó un análisis económico siguiendo la metodología de CIMMYT, para lo cual se consideraron diferentes costos, rendimientos $\mathrm{y}$ beneficios. Se tomaron los datos de rendimientos promedio $(\mathrm{R} \chi)$ por tratamiento y se obtuvo el rendimiento ajustado (Rajust $=10 \%$ de $\mathrm{R} \chi$ ), luego se calculó el beneficio bruto multiplicando el Rajust por el precio de venta de campo USD 0.48 el kilo de tomate. Para la sumatoria de los costos totales que varían, se estimó los costos de los insecticidas evaluados más el costo de aplicación de cada uno de ellos. Para obtener los costos fijos se incluye la depreciación de equipos usados, costos de insumos usados, mano de obra, control de plagas y enfermedades etc.

Análisis de dominancia. Este análisis de dominancia se efectuó ordenando los costos variables de cada tratamientos de menores a mayores, se dice que un tratamientos es dominado cuando sus beneficios netos son menores o iguales a los de un tratamiento que tiene costos que varían más bajos.

Tasa de retorno marginal (TRM). Es un procedimiento que se utiliza para calcular las tasas de retorno marginal entre los tratamientos no dominados, iniciando con el tratamiento de menor costo y procediendo paso a paso a los que les siguen en escala ascendente. Se calculó mediante la fórmula:

TRM $=$ Beneficio marginal $\div$ Costo marginal $\times 100$

\section{RESULTADOS Y DISCUSIÓN}

Fluctuación poblacional de (Bemisia tabaci) por efecto de los tratamientos químicos y el testigo. Se comparó la fluctuación poblacional de mosca blanca en parcelas de tomate tratadas con Engeo, Imidacloprid y el Testigo desde noviembre 25 del 2010 a enero 27 del 2011 (figura 1). Se observó que las poblaciones de mosca blanca se presentaron a partir de la primera fecha de muestreo en noviembre 25 en todos los tratamientos. Se observa en la figura 1 que se presentaron dos picos poblacionales en las fechas diciembre 30 y enero 27. En la fecha de diciembre 30, los mayores promedios de mosca blanca se presentaron en el tratamiento Testigo con 4.75, seguido del tratamiento Engeo con 4.13 y el tratamiento Imidacloprid con 3.13. En la fecha enero 27 los mayores promedios se presentaron en el tratamiento Engeo con 26.69 seguido del tratamiento Imidacloprid con 22.9 y los menores promedios se registraron en el tratamiento Testigo con 18.18 moscas. Al realizar el análisis de varianza, este muestra que existe diferencia significativa $(\mathrm{P}=0.0002)$ entre los tratamientos, donde el Testigo obtuvo el promedio más bajo con 2.35 moscas blancas por planta, seguido por el tratamiento Imidacloprid con 2.44 y Engeo con 2.64 mosca blancas por planta. En este estudio es importante aclarar que 
el muestreo de mosca blanca se realizó desde las 7:30 de la mañana hasta la una de la tarde. Este tiempo de más de 5 horas de muestreo, fue debido a que el número de plantas muestreadas fue bien alto (600 plantas en todo el ensayo), y a medida que las plantas iban creciendo, el muestreo de mosca

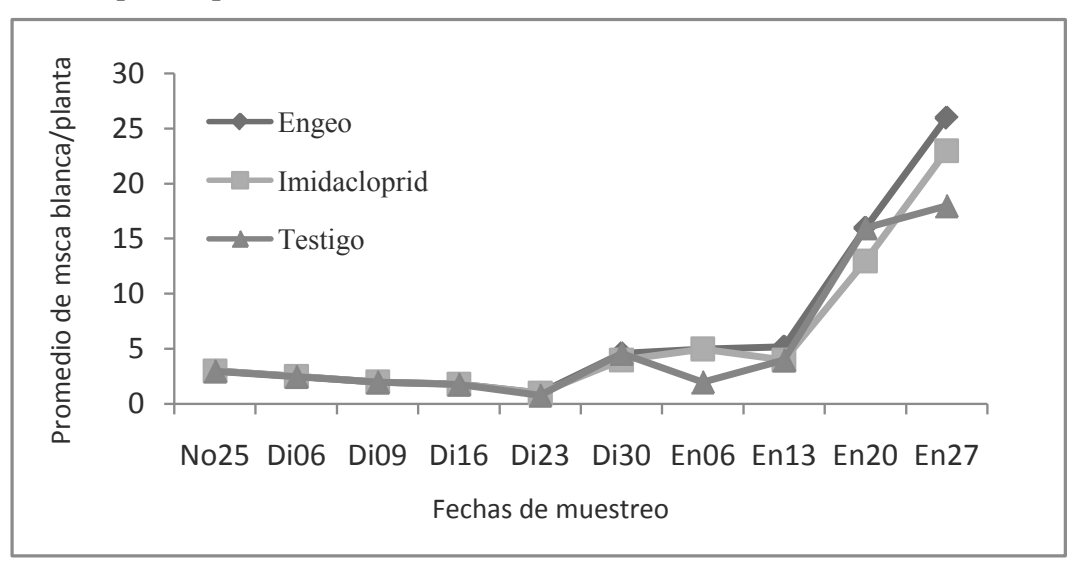

Figura 1: Fluctuación poblacional de mosca blanca en los tratamientos químicos y testigo, noviembre 2010 a enero 2011, Tisma, Masaya.

blanca era más tardado. El último tratamiento muestreado fue el testigo, esto pudo haber enmascarado los resultados, ya que al mediodía las poblaciones de mosca blanca eran ya menores comparado con las horas tempranas de la mañana.

Fluctuación poblacional de (Bemisia tabaci) por efecto de los tratamientos botánicos y el testigo. Se comparó la fluctuación poblacional de mosca blanca en parcelas de tomate tratadas con Crisantemo, Madero Negro, Chile+Ajo+Jabón y Testigo desde noviembre 25 del 2010 hasta enero 27 del 2011 (figura, 2). Se observó que las poblaciones de moscas blancas se presentaron a partir de la primera fecha de muestreo, noviembre 25 para todos los tratamientos. Se observa en la figura 2 que se presentaron tres picos poblacionales en las fechas enero 06 , enero $20 \mathrm{y}$ enero

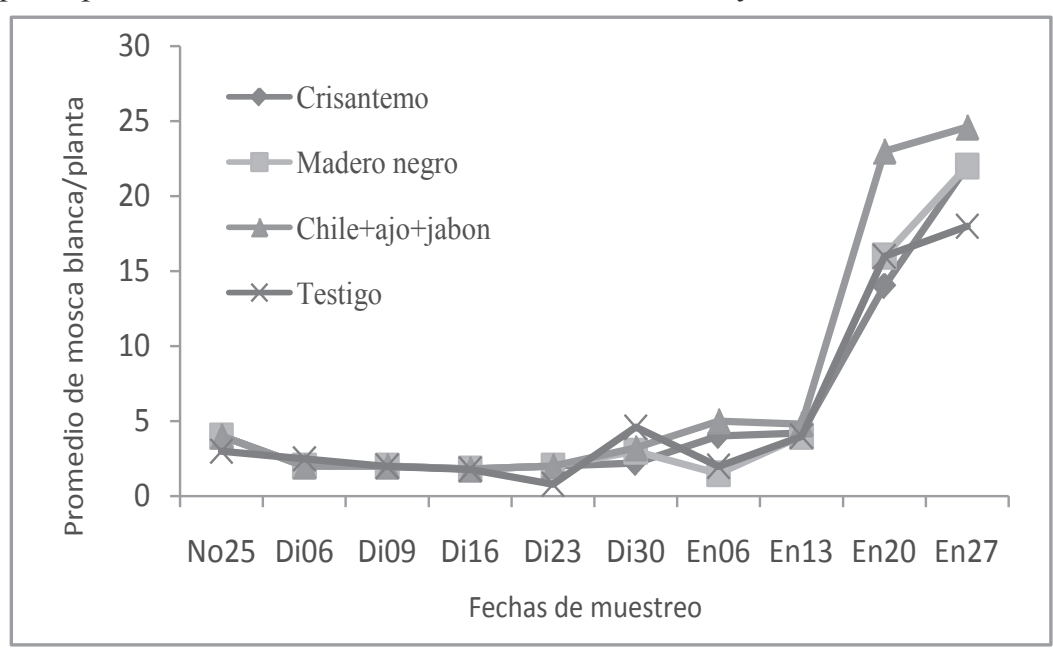

Figura 2. Fluctuación poblacional de mosca blanca, en los tratamientos botánicos y Testigo, noviembre 2010 a enero 2011, Tisma, Masaya.
27. En la fecha enero 06, los menores promedios de mosca blanca se presentaron en los tratamientos Testigo, Madero Negro y Crisantemo con 1.64, 1.64 y 4.27 moscas blancas, en la fecha enero 20 los menores promedios se presentaron en el tratamiento Crisantemo con 13.11, seguido del tratamiento Testigo con 16.25 y Madero Negro con 17.57 y el tratamiento Chile+Ajo+Jabón presentó el mayor promedio con 22.22 , en la fecha enero 27 los menores promedios se presentaron en el tratamiento Testigo con 18.18 y el tratamiento que presenta el mayor promedio fue Chile+Ajo+Jabón con 24.12 en comparación con el resto de los tratamientos. El análisis de varianza de la fluctuación poblacional de mosca blanca indica que hay diferencia significativa $(\mathrm{P}=0.0002)$ entre los tratamientos, donde el tratamiento Testigo refleja los promedios más bajos con 2.35 moscas blancas por planta seguido de Crisantemo con 2.44 moscas blancas por planta, Madero Negro con 2.51 moscas blancas por planta y el promedio más alto lo obtuvo Chile+Ajo+Jabón con 2.69 moscas blancas por planta.

La incidencia de mosca blanca se presentó, alimentándose del envés de las hojas, desde la primera fecha de muestreo, noviembre 25 hasta enero 27. La mosca blanca presentó la mayor incidencia en la fecha de enero 27 , en este pico el tratamiento que presentó menor promedio fue el Testigo en comparación con los demás tratamientos evaluados, y el tratamiento que presentó mayor incidencia de mosca blanca fue el Engeo. El análisis de varianza demuestra que existen diferencias significativas entre los tratamientos evaluados. La presencia de mosca blanca en plantaciones de tomate es inevitable debido a que es su plaga principal, sus poblaciones aumentan en la medida que se utilizan insecticidas de amplio espectro, lo que provoca la resistencia del insecto a largo plazo al hacer mucho uso de insecticidas sistémicos, esto ocasionaría el desarrollo de resistencia del insecto. Al realizar comparaciones con estudios similares en el municipio de Tisma, Masaya donde se evaluaron cinco alternativas de manejo sobre la incidencia del complejo mosca blancaGeminivirus en tomate en etapa de campo, en el cual se evaluaron tres de los tratamientos usados en esta investigación (Engeo, Madero Negro y Chile+Ajo+Jabón. El Madero Negro resultó ser uno de los que mejor controlaron mosca blanca, seguido de Chile+Ajo+Jabón (Cerda, 2011). Según estudios realizados en el municipio de Tisma, Masaya donde se evaluaron cuatro alternativas de protección físicas y químicas de semilleros de tomate contra el ataque del complejo mosca blanca-Geminivirus y su efecto en el rendimiento, se encontraron poblaciones 
mínimas de 2.15 moscas blancas por planta, estos resultados coinciden con los encontrados en este estudio, ya que se encontraron poblaciones mínimas de 2.35 moscas por planta (Rodríguez y Morales, 2007). En Nicaragua la mayor incidencia de mosca blanca se presenta en la estación seca y muestra un incremento durante la canícula que se presenta del 15 de julio al 15 de agosto, registrándose picos máximos de incidencia de moscas blancas a finales de febrero (CATIE, 1990). En este estudio se observó que durante las primeras cuatro fechas de muestreo desde noviembre 25 a diciembre 16 del 2010, las poblaciones de mosca blanca fueron bajas, después las poblaciones se incrementaron desde la fecha de diciembre 23 del 2010 hasta el 27 de enero 2011. El incremento de las poblaciones de mosca blanca a partir de diciembre 23, probablemente se deben al cambio de estación climática del invierno a verano, lo cual crea condiciones favorables para que las poblaciones de moscas blancas aumenten (CATIE, 1990), (Hilje, 2000).

\section{Incidencia (\%) de virosis trasmitido por (Bemisia tabaci) en los} tratamientos químicos y testigo. Se comparó el porcentaje de incidencia de daño de virosis transmitido por mosca blanca en los tratamientos Engeo, Imidacloprid y Testigo en el cultivo del tomate desde diciembre 23 del 2010 a enero 27 del 2011. Se observa en la figura 3 que se presentaron tres picos importantes de incidencia del daño de virosis transmitido por mosca blanca siendo estos en diciembre 23, enero 13 y enero 27. En la fecha diciembre 23 el menor porcentaje de incidencia del daño de virosis se presentó en el tratamiento Imidacloprid con 24\%, seguido del tratamiento Engeo con 35\% y la mayor incidencia se presentó en tratamiento Testigo con $41 \%$. En la fecha enero 13 el tratamiento Imidacloprid mantiene la misma incidencia del daño de virosis y el tratamiento Testigo presentó menor incidencia de la virosis, en la fecha enero 27 el menor porcentaje de incidencia del daño de virosis se presentó en los tratamientos Engeo e Imidacloprid con 49\% y 54\%, el mayor porcentaje lo presentó el tratamiento testigo con 57\%. El análisis de varianza indica que existe diferencia significativa $(\mathrm{P}=0.04)$ entre los tratamientos evaluados, donde los tratamientos que presentaron el menor porcentaje de incidencia a lo largo de todo el ciclo del cultivo fueron Imidacloprid con $28.66 \%$ y el mayor porcentaje de incidencia lo presentó Engeo con $37.16 \%$ y Testigo con $37.66 \%$.
Incidencia (\%) de virosis trasmitido por (Bemisia tabaci) en los tratamientos botánicos y testigo. Se comparó el porcentaje de incidencia del daño de virosis transmitido por mosca blanca en los tratamientos Crisantemo, Madero Negro, Chile+Ajo+Jabón en comparación con el Testigo en el cultivo del tomate desde diciembre 23 a enero 27. Se observa en la figura 4 que se presentaron tres picos de incidencia del daño de virosis transmitido por mosca blanca en diciembre 30 , enero 13 y enero 27. En la fecha diciembre 30 el menor porcentaje de incidencia se presentó en el tratamiento Testigo con $27 \%$, seguido del tratamiento Chile+Ajo+Jabón con $32 \%$ y el mayor porcentaje de incidencia lo presentó el tratamiento Crisantemo con 41\%. En la fecha enero $13 \mathrm{el}$ menor porcentaje de incidencia lo presentó el tratamiento

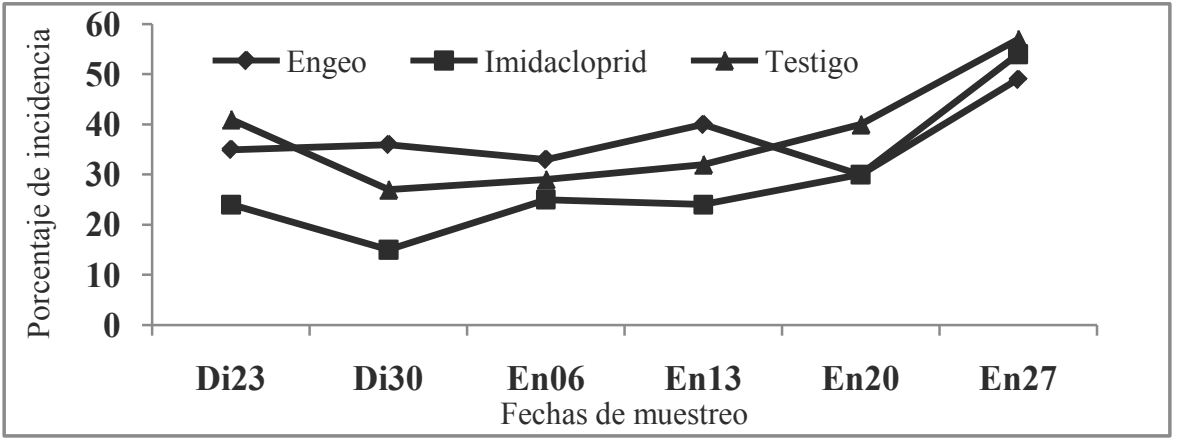

Figura 3. Porcentaje de incidencia del daño de virosis transmitido por mosca blanca en los tratamientos químicos y Testigo, diciembre 2010 a Enero 2011, Tisma, Masaya.

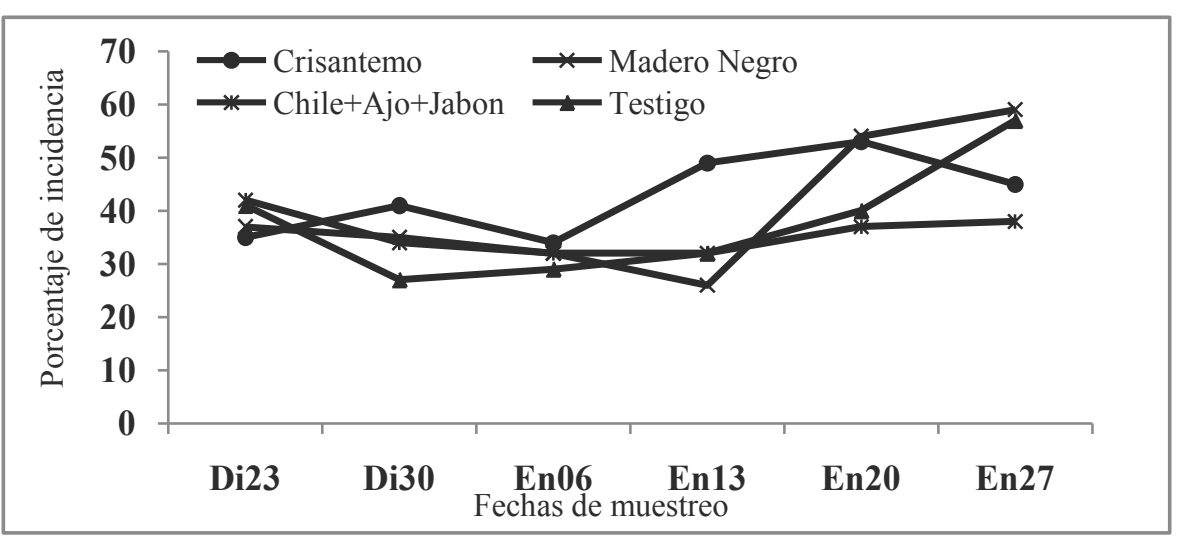

Figura 4. Porcentaje de incidencia del daño de virosis transmitido por mosca blanca en los tratamientos botánicos y testigo, diciembre 2010 a enero 2011, Tisma, Masaya.

Madero Negro con 26\%, y el mayor porcentaje lo presentó el tratamiento Crisantemo con $49 \%$. En la fecha enero 27 el menor porcentaje de incidencia del daño de virosis lo presentó Chile+Ajo+Jabón con 38\% y el mayor porcentaje lo presentó el tratamiento Madero Negro con 59\%. El análisis de varianza realizado indica que existe diferencia significativa $(P=0.04)$ entre los tratamientos evaluados donde el tratamiento que presentó menor porcentaje de incidencia fue Chile+Ajo+Jabón con $35.83 \%$ y el mayor lo presentó Crisantemo con $42.83 \%$. 
Porcentaje de severidad del daño de virosis trasmitido por (Bemisia tabaci) en los tratamientos químicos y testigo. Se comparó el porcentaje de severidad del daño de virosis transmitido por mosca blanca en los tratamientos Engeo, Imidacloprid en comparación con el Testigo en el cultivo del tomate desde diciembre 23 del 2010 a enero 27 del 2011. Se observa en la figura 5 que se presentaron tres picos diciembre 30 , enero 13 y enero 27 . En la fecha diciembre 30 el mayor porcentaje de severidad del daño de virosis se presentó en el tratamiento Engeo con $10.2 \%$ y el menor porcentaje lo presentó Imidacloprid con $4.2 \%$, en la fecha enero 13 el mayor porcentaje de severidad del daño de virosis lo presentó Testigo con $14.4 \%$ y el menor lo presentó Imidacloprid con $9.4 \%$. En la fecha enero 27 el mayor porcentaje de severidad del daño de virosis lo presentó el tratamiento Testigo con $21.8 \%$ y el menor fue Engeo con $16.2 \%$. El análisis de varianza indica que existe diferencia significativa ( $P$ $=0.04$ ) entre los tratamientos, donde el tratamiento Imidacloprid refleja el menor porcentaje de severidad con 9.43\% seguido por Engeo con $10.93 \%$ en comparación con el testigo que presentó $14.06 \%$.

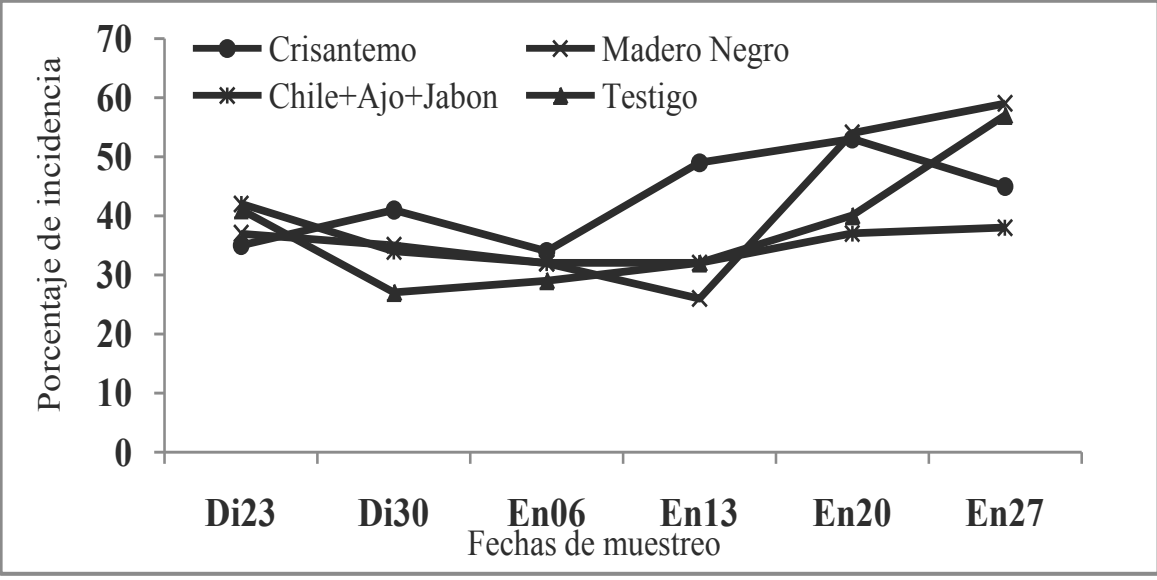

Figura 5. Porcentaje de severidad del daño de virosis transmitido por mosca blanca, en los tratamientos químicos y testigo, diciembre 2010 a enero 2011, Tisma, Masaya.

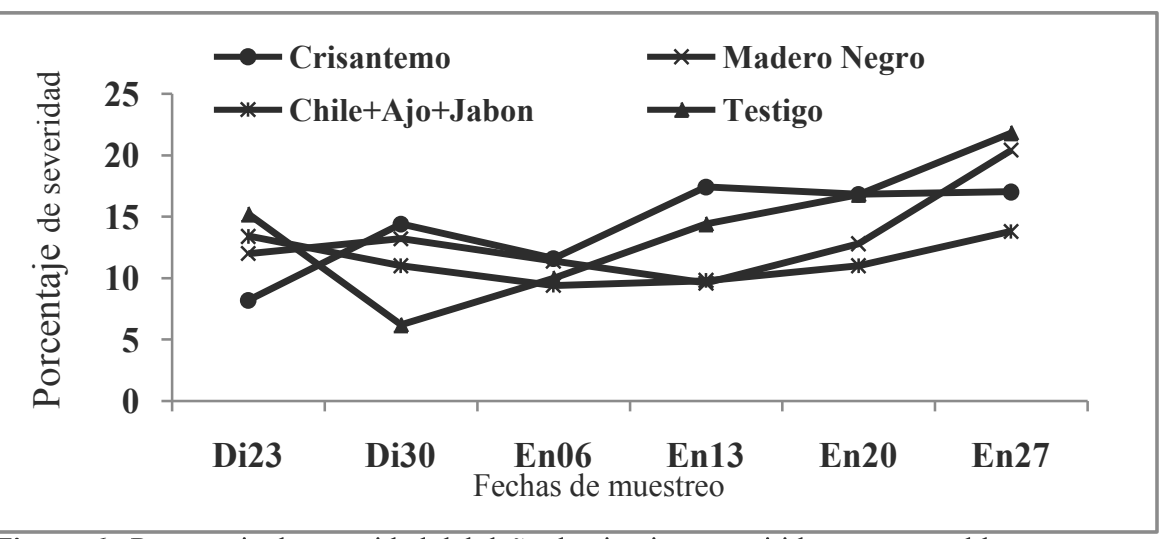

Figura 6. Porcentaje de severidad del daño de virosis transmitido por mosca blanca, en los tratamientos botánicos y testigo, diciembre 2010 a enero 2011, Tisma, Masaya.
Porcentaje de severidad de daño de virosis transmitido por (Bemisia tabaci) en los tratamientos botánicos y testigo. Se comparó el porcentaje de severidad del daño de virosis transmitido por mosca blanca en los tratamientos Crisantemo, Madero Negro, Chile+Ajo+Jabón y Testigo en el cultivo del tomate desde diciembre 23 del 2010 a enero 27 del 2011. Se observa en la figura 6 tres picos en diciembre 30 , enero 13 y enero 27 . En la fecha diciembre 30 el mayor porcentaje de severidad del daño de virosis lo presentó Crisantemo con $14.4 \%$ y el menor porcentaje lo presentó el tratamiento Testigo con $6.2 \%$. En la fecha enero 13 el mayor porcentaje de severidad del daño de virosis lo presentó Crisantemo con $17.4 \%$ y el menor porcentaje lo obtuvo Madero Negro con $9.8 \%$, En la fecha enero 27 el mayor porcentaje de severidad del daño de virosis lo presentó el tratamiento Testigo con $21.8 \%$ y el menor porcentaje lo obtuvo el tratamiento Chile+Ajo+Jabón con 13.8\%. El análisis de varianza indica que existe diferencia significativa $(\mathrm{P}=0.04)$ entre los tratamientos, donde el tratamiento Chile+Ajo+Jabón presentó el menor porcentaje de severidad con $11.4 \%$, seguido por Madero Negro con $13.23 \%$ y el Testigo que obtuvo $14.06 \%$.
Severidad del daño de virosis transmitido por (Bemisia tabaci) a los 93 ddt. Se comparó la severidad del daño de virosis transmitido por mosca blanca a los $93 \mathrm{ddt}$, se observó que los mayores porcentajes de severidad del daño de virosis lo presentaron los tratamientos Testigo, Madero Negro e Imidacloprid con $21.8,20.4$, y $17.2 \%$, en cambio los menores porcentajes de severidad de daño de virosis se presentó en los tratamientos Chile+Ajo+Jabón, Crisantemo y Engeo con 13.8, 17 y 16.2\%, indicando que existen diferencias significativas entre los tratamientos.

Los síntomas de virosis transmitido por $B$. tabaci se caracterizan por un amarillamiento general de la planta afectada, al que se suma un enanismo marcado, seguidamente de un arrugamiento severo de las hojas terminales de la planta (Hilje y Arboleda, 1992). Los niveles de infestación de virosis en tomate no siempre dependen de la cantidad de adultos por planta, existen estudios donde se puede comparar que los niveles de incidencia y severidad de virosis fueron relativamente similares con poblaciones diferentes de moscas blancas. (Quiroz, et al., 1994), observaron que las mayores poblaciones de $B$. tabaci aceleran la epidemia al desplazar el virus más rápidamente, por otro lado se afirma que pocos 
adultos de $B$. tabaci pueden diseminar la virosis rápida y eficientemente (Hilje, 1993).

El porcentaje de incidencia empieza a reflejarse a partir de diciembre 23 hasta enero 27, siendo en enero 27 cuando se presentaron los picos más altos durante todo el ciclo del cultivo. Los tratamientos que presentan el porcentaje más bajo de incidencia fueron Imidacloprid, Chile+Ajo+Jabón y Madero Negro, mientras que el tratamiento Crisantemo presentó el mayor porcentaje de incidencia de virosis al ser comparado con el Testigo. El porcentaje de severidad del daño de B. tabaci se empieza a observar a partir de diciembre 23 del 2010 hasta enero 27 del 2011. Los tratamientos que presentaron el menor porcentaje de severidad en la fecha enero 27 fueron: Chile+Ajo+Jabón, Engeo, Crisantemo y Imidacloprid, por el contrario, el tratamiento con el más alto porcentaje de severidad resultó ser Madero Negro en comparación con el Testigo. Se puede deducir que la mosca blanca no fue tan incidente ni tan severa debido a los muestreos realizados semanalmente, y a la aplicación oportuna de los productos dirigidos al manejo de dicha plaga sumado a que la variedad utilizada en el estudio es tolerante a la virosis. Por otra parte, las plántulas fueron protegidas en condiciones de microinvernadero durante una de las etapas más críticas del cultivo (semillero), obteniendo así plantas sanas, fuertes y vigorosas.

Los altos porcentajes de severidad en el Testigo se estima es debido a que las aplicaciones consistían solamente en agua, y al momento de aplicar el agua las moscas se van pero existen tendencias que mosca blanca regrese y continúe con el ciclo de infección, no así en el resto de los tratamientos.

En el caso que se presenten altas poblaciones de $B$. tabaci aumenta la severidad del daño de virosis, teniendo como consecuencia altos porcentajes de severidad al punto de alcanzar los grados 3 y 4 de la escala propuesta por JimenezMartinez 2006 y modificada en 2010 (tabla 1). En un estudio realizado en Tisma, Masaya se reportan porcentajes máximos de incidencia del daño de virosis transmitido por mosca blanca de $13.2 \%$ para Chile+Ajo+Jabón y mínimos de $1.9 \%$ para el tratamiento Engeo (Cerda 2011). En cambio en este estudio el tratamiento que presentó el menor porcentaje del daño de virosis transmitido por mosca blanca fue Imidacloprid con $28.66 \%$ y el máximo porcentaje lo obtuvo Crisantemo con $42.82 \%$. Con respecto a la severidad, Cerda (2011), refleja que el tratamiento Chile+Ajo+Jabón presentó el mayor porcentaje de severidad y el menor lo presentó el tratamiento Aceite vegetal+Jabón líquido, por lo que ambos resultados son totalmente diferentes. Otro estudio refleja que Gaucho o Imidacloprid presentó el máximo porcentaje de incidencia y severidad con $56 \%$ y $37 \%$ respectivamente (Rodríguez y Morales 2007). En este estudio, Imidacloprid fue el tratamiento que presentó el mínimo porcentaje de incidencia y severidad del daño de virosis transmitido por mosca blanca con $28.66 \%$ y $9.43 \%$ respectivamente. El porcentaje de incidencia de daño de virosis transmitido por mosca blanca en este estudio se considera bajo en comparación con el estudio realizado por (Rodríguez y Morales, 2007) y otros estudios realizados anteriormente.

Rendimiento total ( $\left.\mathrm{kg} \mathrm{ha}^{-1}\right)$. Se comparó el rendimiento total en $\mathrm{kg} \mathrm{ha}^{-1}$ de las parcelas de tomate en los tratamientos evaluados de noviembre 2010 a enero 2011. Los rendimientos totales obtenidos muestran que el tratamiento que obtuvo el mayor rendimiento fue la parcela tratada con Crisantemo con $32578 \mathrm{~kg} \mathrm{ha}^{-1}$. Los tratamientos tratados con Engeo, Chile+Ajo+Jabón y Madero Negro obtuvieron rendimientos de 31750,30625 y $27313 \mathrm{~kg} \mathrm{ha}^{-1}$ respectivamente, en cambio los tratamientos Imidacloprid y testigo obtuvieron los menores rendimientos con 19625 y $20413 \mathrm{~kg} \mathrm{ha}^{-1}$ respectivamente.

Los tratamientos evaluados en este estudio reflejan que Crisantemo fue el tratamiento que obtuvo el mayor rendimiento en $\mathrm{kg} \mathrm{ha}^{-1}$ con respecto a los demás, seguido de los tratamientos Engeo, Chile+Ajo+Jabón, Madero Negro y los tratamientos que presentaron el menor rendimiento fueron Imidacloprid y Testigo. Cerda (2011), encontró que el tratamiento Engeo y Madero Negro presentaron los mejores rendimientos; esto coincide con los resultados encontrados en este estudio ya que el tratamiento Engeo y Madero Negro se ubican como uno de los mejores tratamientos con respecto al rendimiento.

La disminución de los rendimientos está relacionada a la incidencia de virosis. Lastra (1993) y Acuña (1992), mencionan que la presencia de virosis puede reducir los rendimientos de tomate hasta en un $100 \%$, especialmente si la infección es adquirida en épocas tempranas de crecimiento vegetativo o en etapa de semilleros.

\section{Comparación económica de los tratamientos evaluados}

Presupuesto parcial. El análisis del presupuesto parcial realizado según la metodología del CIMMYT, determinó que los mayores costos variables los obtuvieron los tratamientos Crisantemo, Imidacloprid y Chile+Ajo+Jabón con 97.29, 48.72 y 32.87 USD ha $^{-1}$, los de menor costos variables fueron los tratamientos Madero Negro, Testigo y Engeo con 10.07, 12.40 y 31.92 USD ha $^{-1}$. El tratamiento que obtuvo el mayor beneficio neto fue Crisantemo con $7546.90 \mathrm{USD}^{-1}$, en cambio el tratamiento que presentó los menores beneficios netos fue el Imidacloprid con 2000.02 USD ha $^{-1}$ (tabla 2).

Los resultados agronómicos que se obtuvieron del experimento de campo fueron sometidos a análisis económico. El propósito es determinar la rentabilidad de los tratamientos en comparación con la práctica común de los productores, o simplemente determinar el tratamiento con mejor retorno económico. Todo tratamiento recomendado en la producción debe ajustarse a los objetivos y circunstancias de los productores. Por lo tanto el proceso de aplicación de este enfoque debe generar una recomendación para los agricultores (CIMMYT, 1988). 
Tabla 2. Presupuesto parcial (USD) para los tratamientos evaluados, Tisma, Masaya, 2010-2011

\begin{tabular}{lrrrrrr}
\hline Concepto & \multicolumn{1}{l}{ Engeo } & Imidacloprid & Crisantemo & Madero Negro & Chile+Ajo+Jabón & Testigo \\
\hline Rendimiento $\left(\mathrm{kg} \mathrm{ha}^{-1}\right)$ & 31750 & 19625 & 32578 & 27313 & 30625 & 20413 \\
Rendimiento $\left(\mathrm{kg} \mathrm{ha}^{-1}\right)$ & & & & & & \\
Ajustado $(10 \%)$ & 28575 & 17663 & 29320 & 24582 & 27563 & 18372 \\
Precio de campo (USD kg-1) & 0.48 & 0.48 & 0.48 & 0.48 & 0.48 & 0.48 \\
Ingreso bruto USD ha & 13716 & 8478 & 14074 & 11799 & 13230 & 8819 \\
Total CV USD ha-1 & 31.92 & 48.72 & 97.29 & 10.07 & 32.87 & 12.40 \\
Beneficio neto USD ha & 7255 & 2000 & 7547 & 5360 & 6768 & 2377 \\
\hline
\end{tabular}

CV: Costos variables, Precio oficial del dólar: 21.94, Precio del producto al momento de la cosecha (0.48 USD $\left.\mathrm{kg}^{-1}\right)$

El análisis de presupuesto parcial refleja que el tratamiento Madero Negro presentó los menores costos variables y el tratamiento Crisantemo los mayores costos variables. Los tratamientos Crisantemo y Engeo fueron los que obtuvieron los beneficios netos mayores, por el contrario, el tratamiento que obtuvo el menor beneficio neto fue Imidacloprid.

Análisis de dominancia. El resultado del análisis de dominancia indica que los tratamientos Testigo, Chile+Ajo+Jabón e Imidacloprid resultaron ser dominados por los tratamientos Madero Negro, Engeo y Crisantemo. Por lo tanto, no fueron incluidos en el análisis de la tasa de retorno marginal dado que si son considerados se obtendrá una tasa de retorno marginal negativa (tabla 3 ).

Tabla 3. Análisis de dominancia

\begin{tabular}{lcrl}
\hline $\begin{array}{l}\text { Tratamiento } \\
\text { Dominancia }\end{array}$ & Costo variable & Beneficio neto \\
\hline Madero Negro & 10.1 & $5360 \mathrm{ND}$ \\
Testigo & 12.4 & $2377 \mathrm{D}$ \\
Engeo & 31.9 & $7255 \mathrm{ND}$ \\
Chile+Ajo+Jabón & 32.9 & $6768 \mathrm{D}$ \\
Imidacloprid & 48.7 & $2000 \mathrm{D}$ \\
Crisantemo & 97.3 & $7547 \mathrm{ND}$
\end{tabular}

$\mathrm{ND}=$ No dominado, $\mathrm{D}=$ Dominado.

Estos tratamientos son considerados dominados ya que presentan menores beneficios netos y mayores costos variables que el resto de los tratamientos, por lo tanto, estos fueron excluidos para la realización del análisis de la tasa de retorno marginal.

Análisis de la tasa de retorno marginal. El análisis de la tasa de retorno marginal refleja que para el control de mosca blanca el mejor tratamiento es el Engeo ya que por cada dólar invertido el agricultor obtiene una tasa de retorno marginal de $8671.80 \%$, es decir que por cada dólar invertido se recupera dicho dólar y 86.71 dólares adicionales, siendo estos beneficios mayores que los que aportan los otros tratamientos. Si se usa Crisantemo para el control de la mosca blanca, por cada dólar invertido se obtiene una tasa de retorno marginal de $447.17 \%$ lo cual equivale a 4.47 dólares adicionales una vez recuperado el dólar invertido (tabla 4).

Tabla 4. Análisis de la tasa de retorno marginal

\begin{tabular}{lccccc}
\hline Tratamientos & CV & CM & BN & BM & TRM (\%) \\
\hline Madero Negro & 10.1 & & 5360 & & \\
Engeo & 31.9 & 21.9 & 7255 & 1895 & 8672 \\
Crisantemo & 97.3 & 65.4 & 7547 & 292 & 447 \\
& & & & & \\
\hline
\end{tabular}

CV: Costo variable, CM: Costo marginal, BN: Beneficio neto, BM: Beneficio marginal, TRM: Tasa retorno marginal.

\section{CONCLUSIONES}

De los tratamientos evaluados, el menor promedio de moscas blancas lo presentó el tratamiento Imidacloprid, seguido de Crisantemo.

El tratamiento Imidacloprid fue quien presentó el menor porcentaje de incidencia y el menor porcentaje de severidad al ser comparado con el resto de los tratamientos evaluados.

El tratamiento que obtuvo mayor rendimiento comercial fue Crisantemo seguido de Engeo y Chile + Ajo +Jabón.

El análisis de dominancia realizado demuestra que los tratamientos Madero Negro, Engeo y Crisantemo resultaron no dominados, siendo estos los mejores tratamientos desde el punto de vista económico.

El análisis de la tasa de retorno marginal indica que el tratamiento Engeo tiene una tasa de retorno marginal de 8 $671.80 \%$ lo que quiere decir que por cada dólar invertido se obtiene una ganancia de 86.71 dólares netos adicionales y si usa crisantemo se obtiene una tasa de retorno de $447.17 \%$ es decir una ganancia de 4.47 dólares netos adicionales. 


\section{AGRADECIMIENTO}

Los autores agradecen a la Sra. Elizabeth González y al Sr. Anuar González, productores de Tisma, Masaya por habernos facilitado su finca para la realización de la investigación.
A la Universidad Nacional Agraria (UNA) y a ASDI (Agencia Sueca para el Desarrollo Internacional) por el financiamiento económico para el estudio.

\section{REFERENCIAS BIBLIOGRÁFICAS}

Acuña, W. 1992. Efecto de la infección de un Geminivirus sobre el rendimiento del cultivo de tomate en diferentes estados de desarrollo de la planta. Tesis Ing. Agr. Turrialba, CR, Universidad de Costa Rica.

Alemán, F. 2004. Manual de investigación agronómica: con énfasis en ciencia de las malezas. Managua, NI. 248 p.

AMUNIC (Asociación de municipios de Nicaragua). 2005. Municipio: caracterización de municipios de Masaya (en línea). Managua, Nicaragua. Consultado el 17 de ago. 2011. Disponible en: http//www.amunic.org/.

Arrivillagas, S; Bautista, J; Loarca, A; Montenegro, R; Yax, O. 1997. Tecnologías campesinas como base para el desarrollo rural del occidente de Guatemala. en memoria VIII congreso Nacional de Manejo Integrado de Plagas. 224 p.

Brown, J. K; Bedford, I. D; Bird, J; Costa, H. S; Frohlich, D. R; Markham, P. G. 1995. Characterization and distribution of esterase electromorphs in the whitefly, Bemisia tabaci (Genn.) (Homoptera: Aleyrodidae). Biochemical Genetics 33: 205- 213.

Caballero, R. 1996. Metodología para el estudio y manejo de mosca blanca y Geminivirus. ed. Hilje. L. Turrialba. Costa Rica. p1-10.

CATIE (Centro agronómico tropical de investigación de enseñanza). 1990. Guía para el manejo integrado de plagas del cultivo del tomate. Turrialba, CR. p. 45.

Cerda C, KJ. 2011. Evaluación de alternativas de manejo contra el complejo mosca blanca (Bemisia tabaci, Gennadius)-Geminivirus en el cultivo del tomate (Lycopersicum esculentum MILL) en Tisma, Masaya (2009) y Camoapa, Boaco (2010).Tesis de MS.c. Managua Nicaragua. Pag. 64.

Chávez, A. 2008. Extractos vegetales con efectos fungicida, insecticida o nematicida. Sistema unificado de información institucional. Ministerio de agricultura y ganadería. CR. 2 p.

CIMMYT (Centro Internacional para el Mejoramiento del Maíz y el Trigo MX). 1998. La formulación de recomendaciones a partir de datos económicos. Un manual metodológico de evolución económica. ME. DF. 79 p.

Domínguez, M. 2000. Control biológico y extracto botánico para el control de plagas y enfermedades. Escuela de estudio de postgrado (MUPLAN) Agroecología de plantas medicinales. Facultad de ciencias químicas y farmacias. Facultad de agronomía, Universidad de San Carlos, GT. Pag. 25.

Gómez, D. 1992. Evaluación de seis variedades de tomate (Lycopersicum esculentum Mill) bajo un manejo MIP para el complejo mosca blanca- Geminivirus, en el valle de Sébaco. NIC. $51 \mathrm{p}$.

Hilje, L. 1993. Un esquema conceptual para el manejo de la mosca blanca. (Bemisia tabaci), en el cultivo del tomate. Manejo integrado de plaga. Turrialba, (Costa Rica). 29:51-57.

Hilje, L. 2000. Use of living ground covers for the managing whitefly Bemisia tabaci as a Geminivirus vector in tomatoes. In Proceedings British Crop Protection Council-Pest y Diseases (2000, Brighton, UK). V. 1. p.167-170

Hilje, L; Arboleda, O. 1992. Las moscas blancas (Homóptera: Aleyrodidae) en América central y el Caribe. Informe técnico No 205. Área de protección. (CATIE). Turrialba, Costa Rica. p.66.

INTA (Instituto Nicaragüense de Tecnología Agropecuaria). 1999. Cultivo del Tomate. Guía Tecnológica. Ed. Henner Obregón, № 22. Managua Nicaragua. p. 55.

INTA (Instituto Nicaragüense de Tecnología Agropecuaria, NI). 2004 manejo integrado de plagas cultivo del tomate. Primera edición. Managua, NI. p 3.

Jarquín, D, 2004. Evaluación de cuatro variedades de tomate (Lycopersicum esculentum Mill). Basado en el complejo mosca blanca (Bemisia tabaci) Geminivirus, en la comunidad de Apompúa, Potosí, Rivas, Nicaragua. Tesis de MS.c. Managua, Nicaragua. p 2125.

Jiménez-Martínez, E. 2006. Guía de manejo integrado de mosca blanca y virus en Nicaragua. Ph.d. Entomología- docente. Investigador UNA, coordinador proyecto UNA-CIAT-mosca blanca. Nicaragua $30 \mathrm{p}$.

Jiménez-Mártinez, E. 2007. Evaluación de alternativas de semillero de tomate (Lycopersicum esculentum Mill) contra el ataque del complejo mosca blanca (Bemisia tabaci, Gennadius).Geminivirus. La Calera No 6. UNA. NI. Pag. 63

Lastra, R. 1993. Los geminivirus: un grupo de fitovirus con características especiales. In. Las moscas blancas (Homóptera: Aleyrodidae) en América Central y el Caribe. Eds. Hilje y O. Arboleda. Turrialba, CR. CATIE. p. 16-19. (Serie Técnica. Informe Técnico No. 205). Pag. 10

MAGFOR (Ministerio Agropecuario y Forestal). 2007. Área cosechada, rendimientos y producción de hortalizas a nivel nacional. Ciclo agrícola de 1999-2005. Managua, NI estudio preliminar. Pag. 12.

Peralta, I.E; Spouner, DM. 2007. History origin and early cultivation of tomato (Solanaceae) pp: 1-27. In genetic improvement of solanaceous crops, Vol.2 tomato.M K. Razdan and A.K. Matoo (eds), science publishers, infields, USA. Pag. 14.

Quiros, CA; Ramírez, O; Hilje, L. 1994. Participación de los productores en adaptar y evaluar tecnologías de semillero contra mosca blanca (Bemisia tabaci), en tomate. Manejo Integrado de Plagas. Turrialba. Costa Rica. p. 1-7.

Rodríguez Salguera, BH; Morales Blandon, JL. 2007. Evaluación de alternativas de protección físicas y químicas de semillero de tomate (Lycopersicum esculentum Mill) contra el ataque del complejo mosca blanca (Bemisia tabaci, Gennadius)-Geminivirus y su efecto en el rendimiento, en el municipio de Tisma, Masaya. Tesis Ing. Agr. Managua, NI. p. 1 - 4 
Rojas, A; Kvarnheden, A; Valconnen J, PT. 2000. Geminivirus infesting tomato in Nicaragua. Plant. Disc. 89. p. 843-846.

Syngenta Sf. Engeo®. (En línea). Consultado el 18 de Agosto del 2011. Disponible en http//www.syngenta.com/country/cl/cl/solución/ proteccióncultivos/documents/etiqueta/engeo.pdf.

Vanderplank, E.J. 1963. Plant diseases: Epidemiology and control. New York. Academic press. 69 p.

Zúñiga, C; Ramírez, P. 2002. Los Geminivirus, patógenos de importancia mundial. Manejo integrado de plagas y agroecológica. Turrialba, CR. p. 25-33. 